arXiv manuscript No.

(will be inserted by the editor)

\title{
Two-color photoionization of calcium using SHG and LED light
}

\author{
Carsten Schuck $^{1, \star}$, Felix Rohde ${ }^{1}$, Marc Almendros ${ }^{1}$, Markus Hennrich ${ }^{1,2}$, Jürgen Eschner ${ }^{1}$ \\ ${ }^{1}$ ICFO-Institut de Ciències Fotòniques, E-08860 Castelldefels, Barcelona, Spain. \\ ${ }^{2}$ Institut fur Experimentalphysik, Universitat Innsbruck, Technikerstrasse 25, A-6020 Innsbruck, Austria
}

October 24,2018

\begin{abstract}
We present a photoionization method to load single ${ }^{40} \mathrm{Ca}$ ions in a linear Paul trap from an atomic beam. Neutral Ca I atoms are resonantly excited from the ground state to the intermediate $4 \mathrm{~s} 4 \mathrm{p}^{1} \mathrm{P}_{1}$-level using coherent $423 \mathrm{~nm}$ radiation produced by single-pass second harmonic generation in a periodically poled $\mathrm{KTiOPO}_{4}$ crystal pumped with an $120 \mathrm{~mW}$ extended cavity diode laser. Ionization is then attained with a high-power light emitting diode imaged to the trap center, using an appropriately designed optical system composed of standard achromatic doublet lenses. The setup simplifies previous implementations at similar efficiency, and it hardly requires any maintenance at all.
\end{abstract}

PACS numbers: $32.80 . \mathrm{Fb}, 32.80 . \mathrm{Rm}$

\section{Introduction}

Trapped ions are considered to be one of the most attractive systems for the implementation of scalable quantum information processing [1]. In particular ${ }^{40} \mathrm{Ca}^{+}$has shown to be a well suited qubit candidate because entangled states of two or more qubits can be prepared, coherently controlled and read out with high fidelity [2]. For practical realizations, calcium ions have the important advantage that all light sources required for cooling and information processing are available as (frequency doubled) diode lasers.

In the typical experimental setup a narrow beam of neutral calcium atoms from an oven is directed through the center of a Paul trap where it is overlapped with the beams of the cooling lasers and the photoionization light sources. The state of the art for ionizing calcium atoms in ion trapping experiments is set by the implementation of a two-photon resonance-enhanced photoionization scheme with diode laser sources at $423 \mathrm{~nm}$

\footnotetext{
* Fax: +34-93-553-4000, E-mail: carsten.schuck@icfo.es
}

and 390nm 3,4. The idea of the scheme, as shown in Fig. 1 is to excite the neutral calcium atoms in the thermal beam resonantly on the $4 \mathrm{~s}^{2}{ }^{1} \mathrm{~S}_{0} \rightarrow 4 \mathrm{~s} 4 \mathrm{p}{ }^{1} \mathrm{P}_{1}$ transition and then further to high-lying Rydberg-states which are subsequently Stark-ionized in the electric field of the ion trap [3,5.

The S-P dipole transition has the broadest linewidth of all transitions in Ca I $(35.4 \mathrm{MHz})$ and can easily be saturated with a laser tuned to resonance [4. To excite the electron from the ${ }^{1} \mathrm{P}_{1}$-state into the continuum above the first ionization limit, a second photon of wavelength $\lambda \leq 389.89 \mathrm{~nm}$ is necessary, as e.g. realized in [6]. However, high ionization probability is also reached by exciting high-lying Rydberg-states $(n \approx 40)$ below the first ionization limit $(\lambda \geq 389.89 \mathrm{~nm})$, which are efficiently ionized by the electric field of the Paul trap 7 .

This technique allows for loading rates of more than 100 ions per second [3. Nevertheless, for experiments with single ions much lower rates are preferable, in order to easily control the number of ions loaded into the trap. This is achieved with low-power photoionization light sources and low-flux atomic beams. Only for trapping of rare isotopes, slightly higher rates may be necessary to beat charge exchange processes with ${ }^{40} \mathrm{Ca}$ from the atomic beam [4].

It was observed that even if the $390 \mathrm{~nm}$ laser is operated below its lasing threshold current, its weak incoherent emission was sufficient to load ions 4 . The efficiency of this process was observed to be independent of the linewidth, as a result of the broad autoionizing resonances in the vicinity of the first ionization limit 8 , 9,10. Although the excitation of Rydberg states is the bottleneck of the scheme, sufficiently high loading rates to trap clouds of various calcium isotopes, e.g. ${ }^{43} \mathrm{Ca}^{+}$, were reached in [11,12] using two high-power LEDs in a double-pass imaging configuration.

Despite the satisfactory performance of the two-photon scheme, its implementation is hindered by the fact that $423 \mathrm{~nm}$ and $390 \mathrm{~nm}$ laser diodes have become unavailable. The lasers used in current experiments originate 


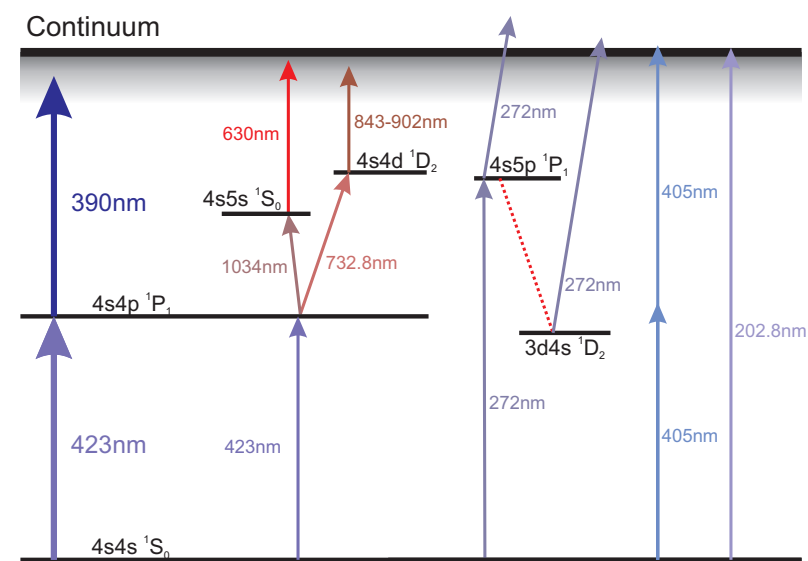

Fig. 1 Selection of realized calcium photoionization schemes: Resonance enhanced excitation of ${ }^{40} \mathrm{Ca}$ to highlying Rydberg states via the $4 \mathrm{~s} 4 \mathrm{p}{ }^{1} \mathrm{P}_{1}$-state [3, 23], resonance enhanced continuum excitation via the $4 \mathrm{~s} 5 \mathrm{p}{ }^{1} \mathrm{P}_{1}$-state 14 and non-resonant (two-photon) excitation of high-lying Rydberg states 19 .

from early production runs of room temperature currentinjection III-IV nitride devices, where the wavelength of the produced diodes varied unpredictably over a range of 380-450nm [13. Meanwhile the leading manufacturers have refined their design and manufacturing techniques and exclusively serve the consumer electronics and optical data storage market with UV laser diodes complying with the Blu-ray disc format (405nm). This situation demands an alternative photoionization scheme for experiments with single ions, which achieves high ionization efficiency and isotope selectivity with reasonable effort and cost.

Previously, photoionization of calcium atoms has also been demonstrated with excitation schemes, e.g. see Fig. 1 which exhibit high efficiency and isotope selectivity but rely on complex and expensive laser systems [14, 15, 16, 17, 18, 19,20]. Apart from these established schemes, an interesting possibility is using a low-cost, free running $405 \mathrm{~nm}$ laser diode for the excitation close to the first ionization limit 21]. For calcium this could be achieved via a non-resonant two-photon transition or direct excitation of high-lying Rydberg states after second harmonic generation of $\lambda \approx 202.5 \mathrm{~nm}$ light, e.g. in $\mathrm{KBe}_{2} \mathrm{BO}_{3} \mathrm{~F}_{2}$ 22].

We pursued a more straightforward approach based on a combination of a frequency-doubled infrared diode laser and a high power LED for a novel implementation of the two-photon resonance-enhanced scheme. To generate narrow-bandwidth $423 \mathrm{~nm}$ radiation, we use a commercially available infrared diode laser and second harmonic generation (SHG) in a nonlinear crystal. This has been demonstrated previously with bulk crystals but due to the restrictions of birefringent phase matching in the UV, sufficient power was only reached with more complex setups involving resonance-enhancement cavities or power amplified master lasers $23,24,25$. Simpler setups for efficient second harmonic generation can be realized with quasi-phase matched materials, i.e. periodically poled crystals [26] or waveguide structures [27. While waveguide structures have higher conversion efficiencies, periodically poled crystals are less expensive and easier to handle in terms of temperature management and pump light coupling.

The experience with a sub-threshold 390nm-laser [4] and light emitting diodes [11,12] also led us to implementing the second excitation step from the ${ }^{1} \mathrm{P}_{1}$-state into the continuum with a high-power LED and a custom designed optical system. The two systems will be described in more detail in the following sections.

\section{Second harmonic generation at $423 \mathrm{~nm}$ in ppKTP}

For the generation of blue and ultraviolet radiation, periodically poled potassium titanyl phosphate (ppKTP) has turned out to be very attractive because stable output can be achieved even at relatively high pump powers [28]. Here we demonstrate how to produce sufficient $423 \mathrm{~nm}$ radiation to saturate the $\mathrm{S}-\mathrm{P}$ transition in $\mathrm{Ca} \mathrm{I}$ by single pass frequency doubling of a single-mode diode laser in a ppKTP crystal. The setup is displayed in figure 2 .

As a pump source we use a grating-stabilized extendedcavity diode laser (Toptica DL100) which has $3-4 \mathrm{MHz}$ linewidth. The SHG radiation will have only half the linewidth of the pump beam [29] and is hence well suited for isotope-selective photoionization. A drawback of using diode lasers for SHG is their elliptical beam shape [28, which reduces the conversion efficiency. To optimize the second harmonic power we therefore use an anamorphic prism pair (see Fig. 2) to achieve a circular collimated beam. We note that the beam shape is a critical parameter for efficient second harmonic generation because only fundamental modes for which the phasematching conditions is fulfilled contribute to the power in the second harmonic.

For a fundamental beam at $\lambda_{\omega}=846 \mathrm{~nm}$ we calculate the poling period $\Lambda=4.05 \mu \mathrm{m}$ in (biaxial) KTP from the Sellmeier equations. Since the second harmonic power increases linearly with crystal length we decided for a crystal of $20 \mathrm{~mm}$ length (crystal dimensions: $20 \times 5 \times 1 \mathrm{~mm}$ ). Taking into account irregularities of the poling period due to imperfect control over the fabrication process, the beam shape of the fundamental light and the high absorption coefficient at $423 \mathrm{~nm}\left(\sim 10 \% \mathrm{~cm}^{-1}[26)\right.$, longer crystals do not necessarily lead to significantly higher single-pass SHG output power.

The crystal 30] is held in a Brass holder, attached to a kinematic prism mount which allows for tilting the crystal entrance surface and hence the optical axis relative to the incoming fundamental beam in horizontal and 


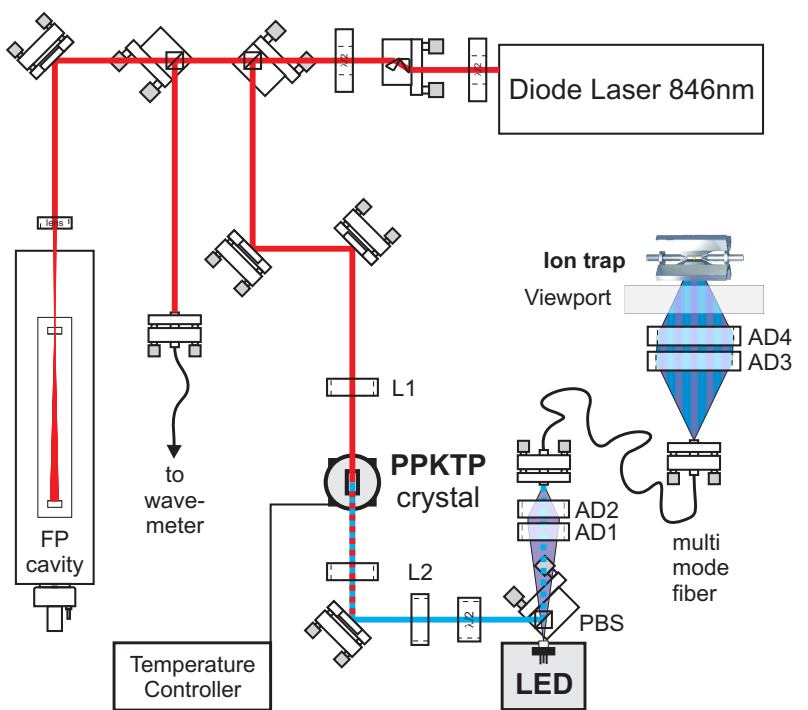

Fig. 2 Setup of photo-ionization light sources. The wavelength and mode behavior of the $846 \mathrm{~nm}$ pump laser are monitored with a wavemeter and a Fabry-Perot (FP) cavity. The temperature of the ppKTP crystal is set and stabilized with a commercial temperature controller (PID-1500, Wavelength electronics). Lenses L1 and L2 are used to adjust the foci of the fundamental $846 \mathrm{~nm}$ beam in the crystal and the SHG $423 \mathrm{~nm}$ beam in the multi-mode fiber, respectively. Achromatic doublets AD1 \& AD2 and AD3 \& AD4 are used to focus both $390 \mathrm{~nm}$ and $423 \mathrm{~nm}$ light into the multi-mode fiber and from there to the trap center, respectively.

vertical direction. To find the optimum trajectory of the focused pump beam through the crystal the holder is mounted on a stack of translation stages allowing us to precisely position the crystal in all three spatial directions.

To maximize the power of the second harmonic we set the focus according to the Boyd-Kleinman theory [31. When scanning the position of the pump beam focus by translating the crystal along the direction of propagation we find a weak position dependence of the second harmonic power which reaches its maximal value at the center of the crystal, as expected 31. To characterize the lateral position dependence we translate the crystal perpendicular to the beam, i.e. horizontally (referred to as $\mathrm{x}$ in Fig 3) and vertically (y). As shown in figure 3, we find that the achievable second harmonic output power depends on the position where the pump beam enters the crystal and is highest close to the top surface. In xdirection, the poling was only applied to the central $3 \mathrm{~mm}$ of the crystal and the edges on either side remained unpoled. The highest SHG conversion efficiency is achieved in the $2500 \times 400 \mu \mathrm{m}$ region below the top surface, Fig. 3] and shows variations of approximately $40 \%$.

These findings are explained by the electric field poling process [32. For our crystal the periodic electrode pattern was deposited on the top surface from where the domains grew in the shape of a needle towards the

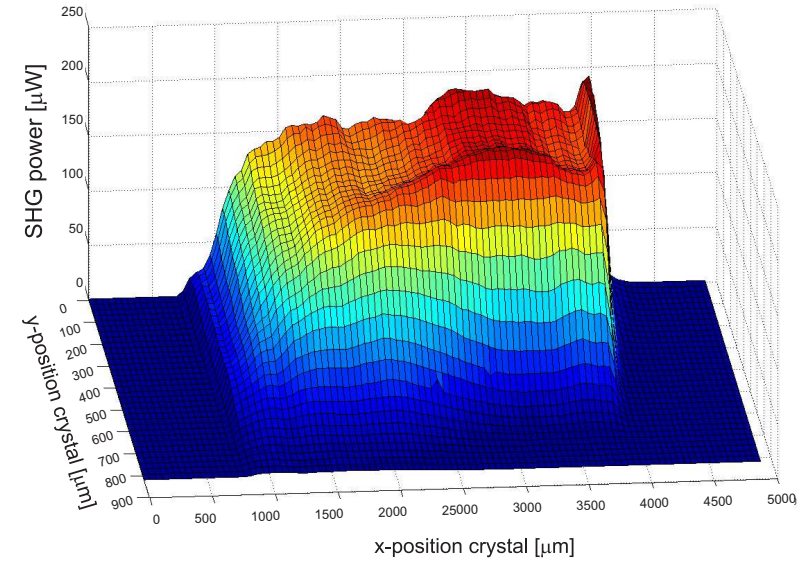

Fig. 3 Single pass SHG output power as a function of the entry position of the pump beam into the ppKTP crystal.

other electrode on the bottom side. Hence, close to the top surface the domains are best defined and highest uniformity of the quasi-phasematching grating is achieved, which makes second harmonic generation most efficient in this region. Deeper inside the crystal the domains spread out, split and may not reach the bottom side. However, it is not straightforward to draw more detailed conclusions about the quality of the periodically poled medium from these measurements because the spectrum of the pump is too narrow [29].

Importantly, efficient second harmonic generation is reached over a large crystal entry surface area (Fig. 3) such that the setup is relatively insensitive to alignment and requires low maintenance in practice.

Since the indices of refraction depend on temperature, one has to be able to precisely control the crystal temperature to fulfill the phasematching conditions for the desired fundamental wavelength. We achieve this with a thermoelectric element glued to the crystal holder. To set and stabilize the crystal temperature with high accuracy we use a commercial PID circuit controlling the current through the thermoelectric element. This temperature controller provides low-noise current and keeps the crystal temperature stable within $5 \mathrm{mK}$ over 24 hours. As sensor input we use a negative temperature coefficient thermistor (NTC) glued to the crystal holder.

To determine the optimal temperature for second harmonic generation to $423 \mathrm{~nm}$, we vary the crystal temperature while monitoring the frequency-doubled output power after a color-filter (BG39, 2mm) eliminating the residual pump light. The maximum power was reached at $19.9^{\circ} \mathrm{C}$, see Fig 4 a convenient working point above the dew point but easily reachable without the need for an oven as in other implementations [26. The obtained full width at half maximum is $1.4^{\circ} \mathrm{C}$, which shows that our simple temperature control system guarantees suf- 
ficient stability for reliable operation with no need for periodic readjustment.

The observed asymmetry in the temperature dependence is due to a spread of the wavevectors in the focused beam, since the phasematching conditions are fulfilled at different temperatures for different wavevector components of the pump beam [33]. For a beam which is not as tightly focused, the phasematching conditions should be approximately constant over the entire crystal length and one expects a sinc ${ }^{2}$ behavior 34 .

The wavelength of the SHG output is set to resonance with the S-P-transition by tuning the fundamental wavelength. As shown in Fig 2 we split off a small fraction of the pump laser to monitor its wavelength by a wavemeter. By setting the angle of the feedback grating of the extended cavity diode laser we vary the pump wavelength until we observe fluorescence on an EMCCD camera from an intense atomic calcium beam through the trap, see Fig. 5]a). This step was performed only once, and the wavemeter reading at maximal fluorescence from the atomic beam is used henceforth as a reference. Before each loading sequence, a slight (piezocontrolled) readjustment of the fundamental wavelength is then sufficient to guarantee efficient excitation of the ${ }^{40} \mathrm{Ca}$ S-P transition. For loading single ions on a daily basis we work with much weaker atomic beams, i.e. lower oven currents, where the atomic fluorescence is hardly visible on the camera.

The Fabry-Perot cavity shown in Fig 2 is used to monitor the (single) mode behavior of the pump laser but could in principle also be used to integrate the pump laser into a transfer-lock frequency-stabilization scheme used for all other laser sources in our experiment [35. This may for example be desirable in experiments where higher isotope selectivity is required. However, it turned out to be unnecessary to do so for experiments with

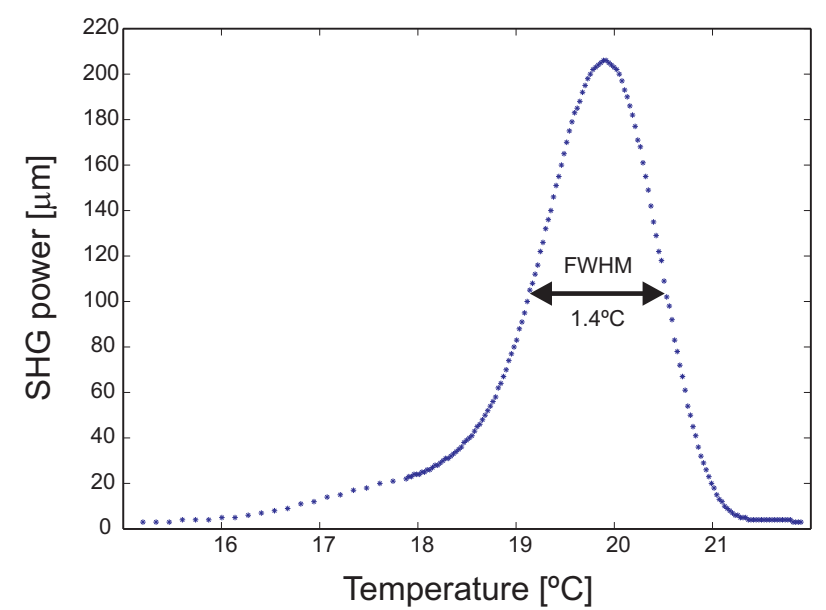

Fig. 4 Temperature dependence of second harmonic generation in ppKTP-crystal at $846 \mathrm{~nm}$. The crystal temperature is controlled with a PID-regulated thermoelectric and varied in steps of $28 \mathrm{mK}$.
${ }^{40} \mathrm{Ca}$. Due to the high intrinsic stability of our external cavity diode laser the drift during loading is relatively small compared to the transition linewidth $(35.4 \mathrm{MHz})$ and the isotope shifts $(>400 \mathrm{MHz})$ 4].

We generate $315.5 \mu \mathrm{W}$ of narrow-bandwidth $423 \mathrm{~nm}$ radiation from a $119 \mathrm{~mW}$ pump beam when all parameters are optimized. This corresponds to a single pass conversion efficiency of $\eta_{\mathrm{eff}}=1.11 \%(\mathrm{~W} \mathrm{~cm})^{-1}$. Taking into account transmission loss in the optical components, the intensity of the $423 \mathrm{~nm}$ light focused to a spot size of $250 \mu \mathrm{m}$ at the trap center reaches approximately $I_{423 \mathrm{~nm}}=5 \mathrm{~mW} / \mathrm{mm}^{2}$, which is sufficient to saturate the S-P-transition of Ca I atoms $\left(I_{\text {sat }}=3.7 \mathrm{~mW} / \mathrm{mm}^{2}\right.$ [4] $)$. Note, that we use a single-frequency laser as compared to previous experiments with, e.g., Ti:sapphire lasers [26], where a large number of modes enhances the conversion efficiency by a factor of two [29. Taking further into account the spatial intensity distribution of the pump beam, our SHG conversion efficiency is comparable to results obtained with significantly more complex setups. In 28, a power amplified single-frequency pump laser and crystals of different length gave similar conversion efficiency values at a different wavelength and slightly higher efficiencies are reported for 423nm SHG in [26], where a larger pump linewidth and a better beam profile were used.

\section{Photoionization with a high-power LED}

Light emitting diodes have shown to be suitable to load ion clouds of different isotopes [1, 12] and single ${ }^{40} \mathrm{Ca}^{+}$ ions 4. For experiments where low loading rates are acceptable, a broad-bandwidth radiation source of relatively low power (i.e. far below saturation of the involved transitions) is sufficient, since many transitions of partially overlapping linewidths and broad autoionizing resonances [18, 36 are available for the (electric field assisted) excitation into the continuum. We chose to use a high-power UV-LED from Nichia (NCCU001) to achieve loading of single ions, and we designed an optical system tailored to the LED emission characteristics. The NCCU001 has significantly higher optical output power than previous UV-LED models, specified between 70 and $85 \mathrm{~mW}$ 37] and does not require a current controller.

The suitability of light emitting diodes for photoionization studies in the past was limited 44 due to their very broad emission spectra, wide emission angles and often large light emitting areas, characteristics which also the NCCU001 displays. Its emission spectrum, centered around $380 \mathrm{~nm}$, has a width of $\Delta \lambda_{\mathrm{FWHM}}=30 \mathrm{~nm}$, of which only a small fraction excites transitions from the ${ }^{1} \mathrm{P}_{1}$-state to high-lying Rydberg states just below the first ionization limit. However, the efficiency of such excitation depends only weakly on linewidth in this wavelength region. The light emitting area is $1 \times 1 \mathrm{~mm}$ large and its angular emission pattern extends over a solid angle of almost $2 \pi$. Hence, the main challenge in using an 
LED instead of a laser diode for photoionization of Ca is to collect and focus its optical output such that sufficiently high intensities are reached within an appropriate volume at the trap center.

For efficient photoionization the atomic beam, the $423 \mathrm{~nm}$ laser and the LED radiation have to overlap in the same volume. We thus consider a spot size of $200 \mu \mathrm{m}$ at the center of the trap as suitable. This is sufficiently small to reach the saturation intensity on the atomic S-P transition with the available $423 \mathrm{~nm}$ power, avoids illumination of the trap electrodes, while covering a large enough part of the atomic calcium beam for the excitation process to be efficient.

To reach high intensity of the LED radiation at the trap center, the optical system has to be optimized for efficient collection from the light emitting surface and for focussing to the desired spot size with low aberrations. The collimation of light from a highly divergent source requires the use of lenses of high numerical aperture. To image the whole LED surface of object size $1 \mathrm{~mm}$ to the envisioned spot size would require an optical system of (de)magnification $m=1 / 5$. This implies that the object distance (LED to optical system) has to be five times larger than the image distance (optical system to demagnified image) and accordingly the numerical aperture (NA) that can be collected from the LED is limited to a fifth of that of the imaging lens. The main restriction on the design is then given by the imaging numerical aperture, which is limited by the $43 \mathrm{~mm}$ distance between the vacuum window and the trap center. Also the UV-transmission properties and aberrations of real lenses impose further restrictions, since high-NA lenses show high spherical and chromatic aberrations.

We performed simulations with an optical system design package (ZEMAX Development Corp.) to find a suitable imaging system based on commercially available, achromatic doublet lenses. Depending on the particular design and the chosen materials available from different manufacturers, aberrations were found to vary significantly but generally become very large for optical systems with numerical apertures $\mathrm{NA} \geq 0.25$ and focal lengths $f \geq 40 \mathrm{~mm}$. We use one inch diameter achromatic doublets (denoted as AD1 and AD2 in Fig. 21) of $f_{\mathrm{AD} 1}=200 \mathrm{~mm}$ and $f_{\mathrm{AD} 2}=40 \mathrm{~mm}$ focal lengths which collect the light emitted into a solid angle of $\Omega \approx$ $0.1 \% \times 2 \pi$ and focus it to a spot size of $215 \mu \mathrm{m}$, corresponding to a magnification $m \approx 1 / 5$. By integrating the LED's angular emission pattern over the solid angle covered by the imaging system and assuming a total horizontally polarized optical output power of $40 \mathrm{~mW}$, we estimate the collected LED power in the whole emitted wavelength range as $P_{c}=210 \mu \mathrm{W}$.

It is useful to couple both light sources into a multimode fiber which is conveniently delivered to the trap, guarantees good spatial overlap of the LED and 423nm beams and occupies minimal optical access to the trap center. We use a multi-mode fiber of $200 \mu \mathrm{m}$ core diame- ter and numerical aperture $\mathrm{NA}=0.22$, which adapts well to the above imaging parameters. To overlap the $423 \mathrm{~nm}$ laser beam and the LED radiation we use a polarizing beam splitter. With lens L2, shown in the setup of Fig. 2, the coupling of both beams to the multi-mode fiber is optimized independently. The one-to-one imaging of the fiber core to the trap center is then realized with achromatic doublets (denoted as AD3 and AD4 in Fig. 2) of $75 \mathrm{~mm}$ focal lengths and $50 \mathrm{~mm}$ diameter. Due to aberrations the actual spot size at the trap center is almost $250 \mu \mathrm{m}$. The optimal distances between the optical components to simultaneously image the $423 \mathrm{~nm}$ laser and the LED's light emitting surface to the fiber input and the fiber output to the trap center is determined from the ZEMAX-simulations. The output fiber coupler and the imaging lenses are mounted on translation stages to overlap the focal spot of the photoionization beams with the atomic beam at the trap center.

To estimate the LED power available for photoionization at the trap center we consider the wavelength range $\lambda=365-391 \mathrm{~nm}$ of the total spectral power distribution, i.e. assuming that Rydberg states down to $n=40$ $(\lambda(n=40) \approx 391 \mathrm{~nm})$ can be ionized in the electric field of the Paul trap. Taking further into account $75 \%$ transmission through the $2 \mathrm{~m}$ long multi-mode fiber, the two achromatic doublet lenses and the $10 \mathrm{~mm}$ thick viewport we obtain $P_{365-391 \mathrm{~nm}} \approx 150 \mu \mathrm{W}$, i.e. an intensity of $I_{365-391 \mathrm{~nm}} \approx 3 \mathrm{~mW} / \mathrm{mm}^{2}$, of which $2.3 \mu \mathrm{W}$ are emitted below the first ionization threshold. A more precise treatment would have to take into account the complex level structure of atoms close to the ionization limit, autoionizing resonances [18,36, series perturbations and core polarization effects 38 .

\section{Loading clouds and single ions}

In our setup the atomic calcium beam is produced from a resistively heated stainless steel tube $(2.5 \mathrm{~mm}$ diameter) filled with granular calcium. To collimate the beam evaporating from this oven we use an orifice of $1 \mathrm{~mm}$ diameter at a distance of $19.8 \mathrm{~mm}$ from the trap center. For trapping single ions we usually work with oven currents of $4.5 \mathrm{~A}$ to $5 \mathrm{~A}$. The cooling lasers, fluorescence collection and detection are described elsewhere [35,39. With the photoionization light sources and the cooling lasers aligned to overlap with the calcium beam at the center of the linear Paul trap, the loading of ions is monitored in real time on an EMCCD camera. We are able to load single ${ }^{40} \mathrm{Ca}^{+}$ions, crystallized ion strings and large ion clouds, see Fig. 5 b)-f).

The linewidth and intensity of the focused $423 \mathrm{~nm}$ radiation are adequate for isotope selective photoionization loading and isotopically pure strings of ${ }^{40} \mathrm{Ca}^{+}$are routinely loaded. The only parameter that has to be adjusted for each loading sequence is the wavelength of the pump laser due to long term drifts. Using the wavemeter, 

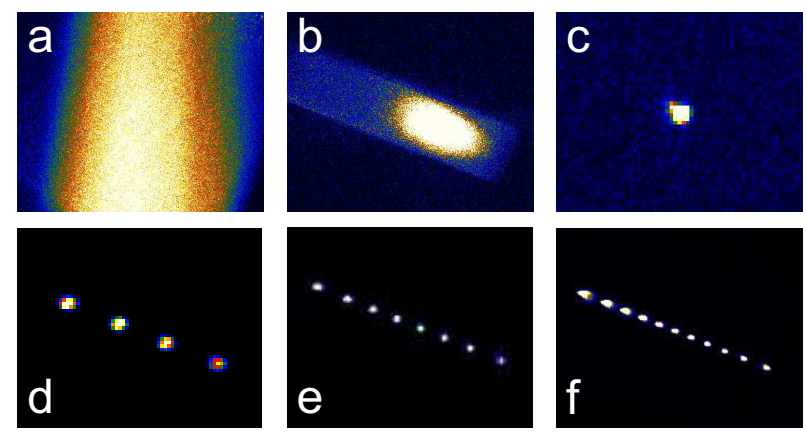

Fig. 5 a) Fluorescence from atomic calcium beam, b) trapped ${ }^{40} \mathrm{Ca}^{+}$cloud seen between the trap electrodes, c) single ${ }^{40} \mathrm{Ca}^{+}$, d)-f) strings of ${ }^{40} \mathrm{Ca}^{+}$.

the setup of the photoionization light sources requires no maintenance on a day to day basis.

While it was not necessary for our application, higher frequency control for isotope-selective S-P excitation may be reached by integrating the monitoring cavity of the $846 \mathrm{~nm}$ pump laser (see Fig. 2) into the transfer locking scheme described in 35. However, different isotopes may also require other laser cooling schemes [4.

The setup we used for photoionization was built with commercially available light sources and off-the-shelf parts and is maintenance-free. The optical system is tailored to the LED emission characteristics and only occupies optical access to the trapping region along one direction. Optimal overlap of the LED and the 423nm SHG laser light at the trap center is achieved by coupling both sources into a multi-mode optical fiber. Our implementation is significantly simpler than those of previous photoionization studies for $\mathrm{Ca}^{+}$trapping experiments, which used a 423nm diode laser, two high-power UV-LEDs and an imaging system occupying optical access along five directions to trap ion clouds [11,12.

\section{Observation of Quantum Jumps}

We note that the LED spectrum is broad enough to not only excite $\mathrm{Ca}$ I atoms from the ${ }^{1} \mathrm{P}_{1}$-state to states in the vicinity of the first ionization limit $(\lambda \approx 389.89 \mathrm{~nm})$, but it also contains spectral components which are resonant with the $\mathrm{S}_{1 / 2}-\mathrm{P}_{3 / 2}$ transition $(\lambda=393 \mathrm{~nm})$ in ${ }^{40} \mathrm{Ca}^{+}$, see figure 6. We observe that the LED power emitted in this frequency range is sufficient to occasionally change the internal quantum state of a laser cooled calcium ion.

Once a single ion or a string of ions is loaded into the trap and the $397 \mathrm{~nm}$ cooling- and 866nm repumplasers are tuned close to resonance with the $\mathrm{S}_{1 / 2}-\mathrm{P}_{1 / 2}$ and the $\mathrm{P}_{1 / 2}-\mathrm{D}_{3 / 2}$ transitions (see Fig. 6), continuous photon scattering is observed. We then switch on the LED again and observe that the resonance fluorescence abruptly ceases to the dark count level and sets in again in irregular intervals on a time scale of around 1s. Such

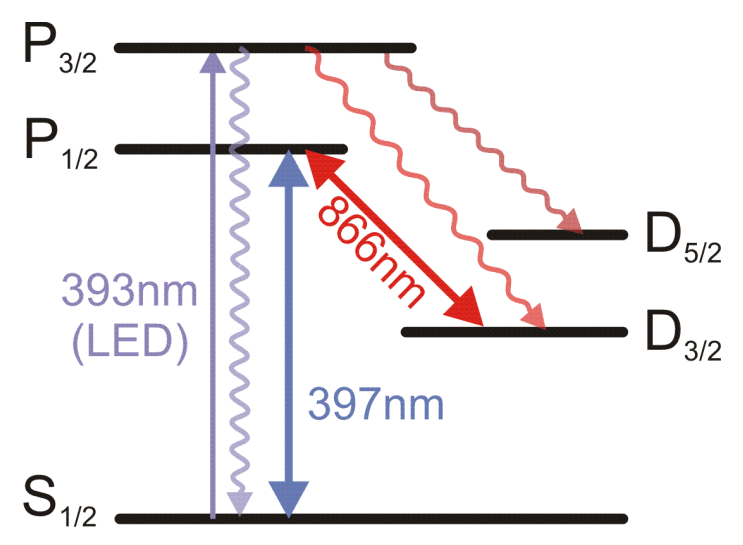

Fig. 6 Level scheme of ${ }^{40} \mathrm{Ca}^{+}$.

random fluorescence extinction occurs when the ion is transferred into a metastable (shelving) state. Similar quantum jumps were first observed in [40,41,42] and demonstrate the quantum nature of resonance fluorescence as they allow for directly monitoring the quantum state of an ion. Thus, the abrupt changes in the fluorescence signal due to jumps to and from a metastable state have practical applications, e.g. for highly efficient detection of atomic transitions or states (electron shelving).

In our case an ion absorbs some of the photons emitted by the LED which are resonant with the $\mathrm{S}_{1 / 2}-\mathrm{P}_{3 / 2}$ transition, see figure 6. Once it is excited to the $\mathrm{P}_{3 / 2^{-}}$ state we distinguish two decay channels. With high probability it will spontaneously decay to the $\mathrm{S}_{1 / 2^{-}}$or $\mathrm{D}_{3 / 2^{-}}$ states from where it reenters the cooling cycle. Since the P-states have very short lifetimes $(\tau \approx 7 n s)$, this detour from the cooling cycle via the $\mathrm{P}_{3 / 2}$-state is not observed in the fluorescence signal. However, spontaneous decay from the $\mathrm{P}_{3 / 2}$-state may also occur, with branching ratio $\Gamma_{\mathrm{D}_{5 / 2}} / \Gamma_{\text {tot }}=1 / 17$, to the metastable $\mathrm{D}_{5 / 2}$-state, which is not part of the cooling cycle. Unlike the P-states, the $\mathrm{D}_{5 / 2}$-state has a long lifetime of roughly one second and the cessation of the fluorescence signal is observable with a CCD-camera or a photodetector, before spontaneous decay from the $\mathrm{D}_{5 / 2}$-state takes the ion back to the $\mathrm{S}_{1 / 2^{-}}$ ground state. From there it reenters the cooling cycle and the fluorescence signal returns to its previous value. The frequency of quantum jumps can be used to optimize the alignment of the photoionization beams to the trap minimum.

We also use the average rate $R_{\mathrm{QJ}} \approx 0.5 \mathrm{~Hz}$ at which we observe quantum jumps on the EMCCD camera to estimate the LED power at the ion in the (non-Gaussian) focal spot area, $A=\pi \omega_{f}^{2}$. For an unpolarized photon resonant with the ionic $\mathrm{S}_{1 / 2}-\mathrm{P}_{3 / 2}$ transition the scattering probability is $p_{\mathrm{sc}}=f_{\mathrm{S}_{1 / 2} \rightarrow \mathrm{P}_{3 / 2}} \cdot \sigma / A$, where $\sigma=$ $\lambda^{2} / 2 \pi$ is the on-resonance cross section and $f_{\mathrm{S}_{1 / 2} \rightarrow \mathrm{P}_{3 / 2}}=$ 0.626 the oscillator strength of the $\mathrm{S}_{1 / 2} \rightarrow \mathrm{P}_{3 / 2}$ transition. From the flux of resonant photons and the photon 
energy we find the power

$$
P_{S P}^{\prime}=\frac{R_{\mathrm{QJ}} \Gamma_{\mathrm{tot}}}{p_{\mathrm{sc}} \Gamma_{\mathrm{D}_{5 / 2}}} \cdot \frac{h c}{\lambda}=14 \mathrm{pW},
$$

A trapped ion thus experiences an intensity of $I_{\mathrm{SP}}^{\prime}=$ $280 \mathrm{pW} / \mathrm{mm}^{2}$ on the $\mathrm{S}_{1 / 2} \rightarrow \mathrm{P}_{3 / 2}$ transition from the LED light field. Using this value to integrate the spectral power distribution numerically over the wavelength range $\lambda=365-391 \mathrm{~nm}$ yields the power available for photoionization at the trap center $P_{365-391 \mathrm{~nm}}^{\prime}=144 \mu \mathrm{W}$, which is in very good agreement with the independently obtained value in section 3 .

\section{Conclusions}

We have implemented a two-photon resonance-enhanced photoionization scheme to trap clouds, strings and single ${ }^{40} \mathrm{Ca}^{+}$ions. We achieve efficient photoionization of calcium atoms in a thermal beam with a high power ultraviolet light emitting diode and single-pass frequency doubling of an infrared extended cavity diode laser in periodically poled KTP, i.e. employing commercially available, inexpensive and robust light sources. With a suitably designed optical system, tailored to the LED emission characteristics and based on off-the-shelf components, we achieve intensities of approximately $I_{423 \mathrm{~nm}}=$ $5 \mathrm{~mW} / \mathrm{mm}^{2}$ and $I_{365-391 \mathrm{~nm}}=3 \mathrm{~mW} / \mathrm{mm}^{2}$ over a spot size of $250 \mu \mathrm{m}$ at the trap center. The setup is simple, maintenance-free, occupies minimal optical access to the trapping region and can easily be adapted to trapping of rare calcium isotopes.

Acknowledgements We acknowledge support by the European Commission (SCALA, Contract No. 015714) and by the Spanish Ministerio de Educación y Ciencia (QOIT, ConsoliderIngenio 2010 CSD2006-00019; QLIQS, FIS2005-08257; QNLP, FIS2007-66944). C.S. acknowledges support by the Commission for Universities and Research of the Department of Innovation, Universities and Enterprises of the Catalonian Government and the European Social Fund.

\section{References}

1. H. Häffner, C. F. Roos, R. Blatt. Phys. Rep., 469:155, (2008).

2. R. Blatt, D. Wineland. Nature, 453:1008, (2008).

3. S. Gulde, D. Rotter, P. Barton, F. Schmidt-Kaler, R. Blatt, W. Hogervorst. Appl. Phys. B, 73:861, (2001).

4. D. M. Lucas, A. Ramos, J. P. Home, M. J. McDonnell, S. Nakayama, J.-P. Stacey, S. C. Webster, D. N. Stacey, A. M. Steane. Phys. Rev. A, 69:012711, (2004).

5. T. R. Gentile, B. J. Hughey, D. Kleppner, T. W. Ducas. Phys. Rev. A, 40:5103, (1989).

6. W. Nörtershäuser, N. Trautmann, K. Wendt, B.A. Bushaw. Spectrochim. Acta B, 53:709, (1998).

7. T. W. Ducas, M. G. Littmann, R. R. Freeman, D. Kleppner. Phys. Rev. Lett., 35:366, (1975).
8. R. W. Ditchburn, R. D. Hudson. Proc. Roy. Soc. (London), A256:53, (1960).

9. V. L. Carter, R. D. Hudson, E. L. Breig. Phys. Rev. A, 4:821, (1971).

10. G. H. Newsom, B. W. Shore. J. Phys. B, 1:742, (1968).

11. U. Tanaka, H. Matsunishi, I. Morita, S. Urabe. Appl. Phys. B, 81:795, (2005).

12. U. Tanaka, I. Morita, S. Urabe. Appl. Phys. B, 89:195, (2007).

13. S. Nakamura, S. Pearton, G. Farsol. Springer-Verlag Berlin Heidelberg New York, (2000).

14. N. Kjærgaard, L. Hornekær, A. M. Thommesen, Z. Videsen, M. Drewsen. Appl. Phys. B, 71:207, (2000).

15. A. Mortensen, J. J. T Lindballe, I. S. Jensen, P. Staanum, D. Voigt, M. Drewsen. Phys. Rev. A, 69:042502, (2004).

16. R. J. Hendricks, D. M. Grant, P. F. Herskind, A. Dantan, M. Drewsen. Appl. Phys. B, 88:507, (2007).

17. L. F. DiMauro, D. Kim, M. W. Courtney, M. Anselment. Phys. Rev. A, 38:2238, (1988).

18. R. S. Dygdała, A. Zawadzka, D. Lisak, P. Płóciennik, R. S. Trawiński. Eur. Phys. J. D, 30:15, (2004).

19. J. A. Armstrong, P. Esherick, J. J. Wynne. Phys. Rev. A, 15:180, (1977).

20. R. Beigang, K. Lücke, A. Timmermann. Phys. Rev. A, 27:587, (1983).

21. K. Vant, J. Chiaverini, W. Lybarger, D. J. Berkeland. arXiv:quant-ph/0607055, (2006).

22. C. T. Chen, T. Kanai, X. Y. Wang, Y. Zhu, S. Watanabe. Opt. Lett., 33:282, (2008).

23. K. Wendt, K. Blaum, B. A. Bushaw, F. Juston, W. Nörtershäuser, N. Trautmann, B. Wiche. Fresenius J. Anal. Chem., 359:361, (1997).

24. C. Degenhardt, T. Binnewies, G. Wilpers, U. Sterr, F. Riehle, C. Lisdat, E. Tiemann. Phys. Rev. A, 67:043408, (2003).

25. C. W. Oates, F. Bondu, R. W. Fox, L. Hollberg. Eur. Phys. J. D, 7:449, (1999).

26. F. Torabi-Goudarzi, E. Riis. Opt. Comm., 227:389, (2003).

27. K. R. Parameswaran, J. R. Kurz, R. V. Roussev, M. M. Fejer. Opt. Lett., 27:43, (2002).

28. J. H. Lundeman, O. B. Jensen, P. E . Andersen, S. Andersson-Engels, B. Sumpf, G. Erbert, P. M. Petersen. Opt. Express, 16:2486, (2008).

29. S. Helmfried, G. Arvidsson. J. Opt. Soc. Am. B, 8:2326, (1991).

30. The ppKTP crystal was supplied by C. Canalias and F. Laurell from the Royal Institute of Technology (KTH), Stockholm, Sweden.

31. G. D. Boyd, D. A. Kleinman. J. Appl. Phys., 39:3597, (1968).

32. C. Canalias, V. Pasiskevicius, A. Fragemann, F. Laurell. Appl. Phys. Lett., 83:734, (2003).

33. G. M. Gibson, G. A. Turnbull, M. Ebrahimzadeh, M. H. Dunn, H. Karlsson, G. Arvidsson, F. Laurell. Appl. Phys. B, 67:675, (1998).

34. H. Karlsson, F. Laurell. Appl. Phys. Lett., 71:3474, (1997).

35. F. Rohde, M. Almendros, C. Schuck, J. Huwer, M. Hennrich, J. Eschner. arXiv:quant-ph/0910.1052, 2009.

36. S. Benec'h, H. Bachau. J. Phys. B, 37:3521, (2004). 
37. In 2008 Nichia replaced the NCCU001 UV LED by the NCSU034A UV LED, which has an LED die of equal size but reaches up $390 \mathrm{~mW}$ optical power output around its peak wavelength of $385 \mathrm{~nm}$ and should hence be even better suited for our application.

38. J. E. Hansen, C. Laughlin, H. W. van der Hart, G. Verbockhaven. J. Phys. B, 32:2099, (1999).

39. S. Gerber, D. Rotter, M. Hennrich, R. Blatt, F. Rohde, C. Schuck, M. Almendros, R. Gehr, F. Dubin, J. Eschner. New J. Phys., 11:013032, (2009).

40. W. Nagourney, J. Sandberg, H. Dehmelt. Phys. Rev. Lett., 56:2797, (1986).

41. T. Sauter, W. Neuhauser, R. Blatt, P. E. Toschek. Phys. Rev. Lett., 57:1696, (1986).

42. J. C. Bergquist, R. G. Hulet, W. M. Itano, D. J. Wineland. Phys. Rev. Lett., 57:1699, (1986). 